\title{
Comparing Banana Leaf Dressing and Vaseline Gauze Dressing for Split-Thickness Skin Graft Donor Sites in a Ugandan Hospital
}

\author{
Naomi Leah Kekisa, George Galiwango, Andrew Hodges \\ Department of Plastic Surgery, Mbarara University of Science and Technology
}

Correspondence to: Dr. Naomi Leah Kekisa; email: naomileahk@gmail.com

Received: 14 November 2019; Revised: 18 April 2020; Accepted: 25 April 2020; Available online: 30 April 2020

\begin{abstract}
Introduction: This study compared the effectiveness of banana leaf dressing (BLD) with the commonly used Vaseline gauze dressing (VGD) on split-thickness skin graft (SSG) donor sites. VGD is not completely nonadherent and is associated with pain on removal. BLD is smooth, non-adherent, pain-free and available. Methods: In this prospective study, consecutive patients were dressed with either BLD or VGD. Ease of applying and removing the dressings was scored. Pain scores were taken on postoperative days 3, 5, 7, 9 and 10 . On day 10 , the dressing was changed, epithelialization recorded and a swab taken for microbial culture. Average cost of each dressing was calculated. Results: There was no significant difference between postoperative pain scores with either dressing $(p=0.992)$. BLD patients had less pain on dressing change $(p=0.006)$. Both dressings were easy to apply; BLD was easier to remove $(p=0.000)$. Wounds with
\end{abstract}

\section{Introduction}

Use of split-thickness skin grafts (SSG) is common in plastic and reconstructive surgeries to cover a wide range of defects (1). This is mainly because of the availability of donor sites (2).

Pain experienced at SSG donor wounds is significant (3) but reduces as the wounds heal. Healing of the donor wound depends on rapid re-epithelialization under moist dressing. The ideal dressing for the SSG donor site should be moist, allow gaseous exchange and remove exudate. It should also be sterile, resistant to infection, comfortable for the patient, easily applied and removed from the wound, and cost effective. (1). Such a dressing
BLD re-epithelialized faster $(\mathrm{p}=0.0158) .40 \%$ of wounds grew no organism on microbial culture, $25 \%$ grew Staphylococcus aureus and $35 \%$ grew unusual organisms $(\mathrm{p}=0.482)$. VGD was 4 times more expensive than BLD ( $p=0.000)$. Conclusion: Banana leaf dressing is effective and highly recommended for dressing SSG donor sites.

Trial registration: PACTR202002762137087

Keywords: Split-thickness skin graft, Donor sites, Banana leaf dressing, Vaseline gauze dressing

Ann Afr Surg. 2021;18(1):4-9

DOI: http://dx.doi.org/10.4314/aas.v18i1.2

Conflicts of Interest: None

Funding: None

(C) 2021 Author. This work is licensed under the Creative Commons Attribution 4.0 International License.

is yet to be developed. Worldwide, practitioners use familiar dressings for SSG donor sites, regardless of performance (1). Vaseline gauze dressing (VGD) or petrolatum impregnated gauze is the dressing most commonly used; however, it is not completely nonadherent and is associated with pain on removal (2). Banana leaf dressing (BLD) has been used as a dressing for surgical wounds in India for three decades now. It is a non-adherent, pain-free dressing. (3). Banana leaf offers a large smooth surface and is cheap and readily available in Uganda throughout the year (4). It has also been found to be a good alternative dressing for surgical wounds in Uganda, in terms of efficiency and cost (5). 


\section{Methods}

\section{Study design}

This was a prospective quasi-randomized controlled study (PACTR202002762137087). This study included all patients 10 years and older who had SSG harvested from the thigh. Patients with comorbidities known to impede normal wound healing, such as diabetes mellitus, peripheral vascular disease, venous insufficiency, among others, and patients with history of substance abuse or long-term pain medication were excluded.

\section{Materials and techniques}

Banana leaf dressing was prepared by removing the midrib, washing with clean water and disinfecting with $0.12 \%$ Chlorhexidine solution. Next, the leaves were divided into $20 \mathrm{~cm}$ by $10 \mathrm{~cm}$ rectangular pieces along the edge of the midrib, packed piece by piece separately into transparent sterilizable pouches and also in Stainless steel Holloware ${ }^{\circledR}$ sterilization drums, and autoclaved at $135^{\circ} \mathrm{C}$ for 15 minutes, and then stored in their respective drums or pouches ready for use. Sterility was confirmed using $3 \mathrm{M}$ Comply ${ }^{\mathrm{TM}}$ SteriGage $^{\mathrm{TM}}$ strips placed into each pouch or drum containing the BLD, according to hospital policy. The VGD was prepared by resizing the gauze and impregnating with Vaseline, packaging in a drum and sterilizing for 2 hours at 135 ${ }^{\circ} \mathrm{C}$. BLD was re-autoclaved every other day while VGD was re-autoclaved daily, as per hospital protocol.

\section{Enrolment of participants}

Eligible patients were identified preoperatively, and consent and assent were obtained. The first participant was selected by tossing a coin and subsequent participant were alternated between each arm of the study. Participants were blinded to the type of dressing applied to their SSG donor site wound.

\section{Intraoperative procedure}

The SSG was harvested under general anesthesia or under spinal anesthesia, using either a Zimmer ${ }^{\circledR}$ Air dermatome or a Humby knife set between 0.5 and 1.0 $\mathrm{mm}$. The choice of dressing for SSG donor sites was made alternately between BLD and VGD for subsequent patients. The dressings were covered with a layer of gauze or Gamgee dressing and crepe bandage. Ease of applying BLD and VGD was scored on a scale of 1 to 10 , and recorded by the nurse or surgeon who applied the dressing.

\section{Postoperative assessment}

Postoperatively, all patients were given Ibuprofen and paracetamol, or drug combinations containing both drugs eight-hourly for 5 days, as per hospital protocol. Patients subjectively scored the donor site pain on days $3,5,7$ and 9 using the $0-10$ numerical pain rating scale (6). On day 10, the dressing was changed and participants scored their donor site pain on dressing change. The ease of dressing removal was recorded by the dressing nurse, on a scale of 1-10. Pictures of the donor site wound were taken with a Canon Powershot SX710 HS digital camera. The percentage surface area of epithelialized wound was recorded as a percentage of the total wound surface area. Two other study-blinded surgeons assessed the photographs of the wounds for percentage of epithelialization. An average of the three values of percentage of epithelialization was then calculated and taken. A wound swab was taken for microbiology culture, according to wound swabbing techniques described by Copper (7). The wound was then redressed until complete healing.

\section{Cost assessment for BLD and VGD}

The cost of producing each material was calculated from the total cost of materials used, labor and time. The unit cost of production was calculated from the total cost divided by the number of pieces of each dressing prepared. The cost of dressing material used for each patient was calculated. A cost analysis compared the cost of using VGD with that of BLD.

\section{Data management and analysis}

Data management and statistical analysis used Epidata 3.1 and STATA11.2.

\section{Ethical approval}

Requisite approval for the study was obtained from CoRSU Hospital, Mbarara University of Science and 
Technology Research and Ethics Committee (MUREC 1/7), National Drug Authority and the Uganda National Council of Science and Technology.

\section{Results}

\section{Participant baseline characteristics}

A total of 95 participants were enrolled in the study. Eighty-six patients (52 (60\%) male and $34(40 \%)$ female) completed the study period successfully while nine were lost to follow-up during the study period. Age ranged from 10 years to 46 years with an average of 18 years; $45(52 \%)$ participants had their donor sites dressed with BLD, while 41(48\%) were dressed with VGD (Table 1). Participants enrolled into the study had various indications for SSG harvest, the commonest being post-burn contracture release (26), next were flap donor site cover (22) and post-traumatic ulcers (12), among other reasons.

\section{Postoperative pain}

Pain scores showed a progressive reduction in pain on SSG donor site wounds with time, using the pain score on day 3 as the baseline. Figure 1 shows no significant relationship between the rates of reduction of pain with the type of dressing used on the wound. Pain was graded as mild (0-3), moderate (4-6) or severe (7-10). Table 1 shows the level of pain participants had upon dressing change on postoperative day 10 . Fewer participants with BLD $(n=11,24 \%)$ experienced severe pain while more participants with VGD experienced severe pain $(n=24$, $59 \%) \mathrm{p}=0.006$ (Table 1 ).

\section{Percentage epithelialization of SSG donor site wounds on postoperative day 10}

Wounds dressed using BLD epithelialized faster than those dressed using VGD, with an average percentage of epithelialization of $81 \%$ by day 10 on wounds dressed with BLD, compared with $69 \%$ for wounds dressed with VGD $(p=0.0158)$ (Table 2$)$.
Figure 1: Average pain scores on different days for BLD and VGD participants

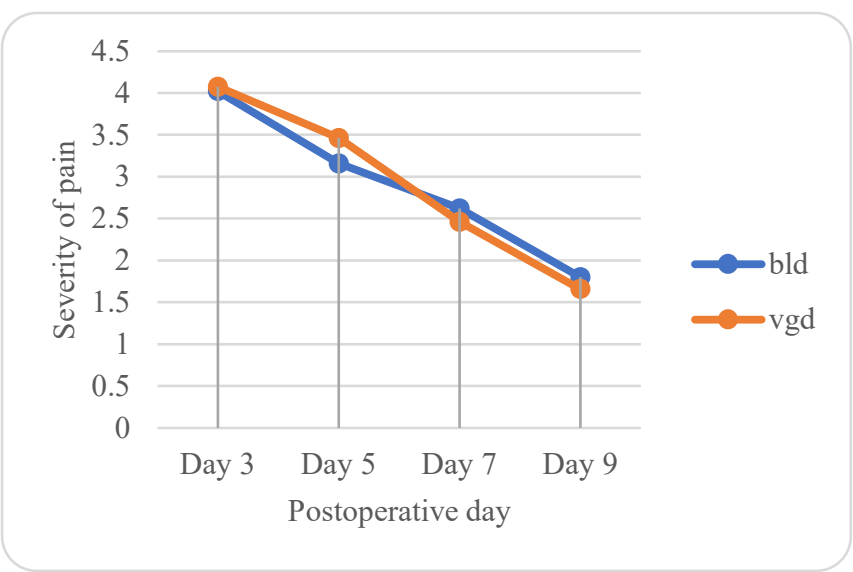

Table 1: Comparison of pain on dressing change with type of dressing

\begin{tabular}{llll}
\hline $\begin{array}{l}\text { Pain on } \\
\text { dressing } \\
\text { change }\end{array}$ & $\begin{array}{l}\text { BLD (\%) } \\
\boldsymbol{n}=45\end{array}$ & $\begin{array}{l}\text { VGD (\%) } \\
\boldsymbol{n}=41\end{array}$ & Total (\%) \\
\hline Mild & $17(37.78)$ & $8(19.51)$ & $25(29.07)$ \\
Moderate & $17(37.78)$ & $9(21.95)$ & $26(30.23)$ \\
Severe & $11(24.44)$ & $24(58.54)$ & $35(40.70)$ \\
\hline Pearson chi $^{2}(2)=10.3665$ & $p=0.006$ &
\end{tabular}

Table 2: Comparison of percentage of epithelialization with type of dressing

\begin{tabular}{|c|c|c|c|c|c|}
\hline $\begin{array}{l}\text { Type of } \\
\text { dressing }\end{array}$ & $n$ & Mean & SD & {$[95 \% \mathrm{CI}]$} & $\begin{array}{l}\mathrm{p} \\
\text { value }\end{array}$ \\
\hline BLD & 45 & 81.44 & 26.17 & $\begin{array}{l}73.58- \\
89.31\end{array}$ & \\
\hline VGD & 41 & 68.98 & 26.67 & $\begin{array}{l}60.56- \\
77.39\end{array}$ & \\
\hline Total & 86 & & & & $\begin{array}{l}\mathrm{p} \quad(\mathrm{T}>\mathrm{t}) \\
=0.0158\end{array}$ \\
\hline
\end{tabular}

Microbial characteristics of SSG donor site wounds

Microbial culture of wound swabs taken from donor site wounds was done. $40 \%$ of the participants had no organism grown on microbial culture. Staphylococcus aureus was the commonest organism grown on culture (29\%) for both participant groups. 19 other types organisms were isolated including Staphylococcus epidermidis, S. haemolyticus, S. microti, Streptococcus pyogenes, Pseudomonas aeruginosa, P. acidovorans, Enterobacter aerogenes, Enterobacter cloacae, Proteus mirabilis, Proteus vulgaris, Morganella morganii, Kluyvera ascorbata, Rahnella aquatilis, Escherichia coli, Erwinia persicina, Hafnia alvei, Yersinia 
KEKISA ET AL.

mollaneti, Citrobacter koseri, Photorhabdus asymbiotica, Photorhabdus luminesce, but none had any significant correlation with the type of dressing used (Table 3).

Table 3: Effect of dressing type on microbial culture

\begin{tabular}{|c|c|c|c|}
\hline Organism identified & BLD (\%) & VGD (\%) & Total (\%) \\
\hline No organism & $14(31.11)$ & $20(48.78)$ & $34(39.53)$ \\
\hline Staphylococcus & $12(26.67)$ & $13(31.71)$ & $25(29.07)$ \\
\hline \multicolumn{4}{|l|}{ aureus } \\
\hline Others & $19(42.22)$ & $8(19.51)$ & $27(31.40)$ \\
\hline Total & $45(100.00)$ & $41(100.00)$ & $86(100.00)$ \\
\hline
\end{tabular}

\section{Usability of dressings}

The usability of dressing was scored subjectively by the surgeons and nurses who applied the dressings, considering ease of application and removal. Usability was scored as easy, moderately easy, or difficult. Table 6 shows that most users found both dressings easy to apply ( $n=84,98 \%)$. Ease of removing dressings was assessed likewise and showed that $96 \%$ of BLD users found it easy to remove with only $4 \%$ finding it moderately easy to remove. Table 4 compares ease of applying and removing the two dressings, $\mathrm{p}=0.000$.

Table 4: Usability of dressings

\begin{tabular}{llll}
\hline Type of dressing & BLD $(\%)$ & VGD (\%) & Total \\
\hline Ease of applying & & & \\
Easy & $43(95.56)$ & $41(100.00)$ & $84(97.67)$ \\
Moderately easy & $1(2.22)$ & $0(0.00)$ & $1(1.16)$ \\
Difficult & $1(2.22)$ & $0(0.00)$ & $1(1.16)$ \\
Ease of removing & & & \\
Easy & $43(95.56)$ & $12(29.27)$ & 55 \\
Moderately easy & $2(4.44)$ & $12(29.27)$ & 14 \\
Difficult & $0(0.00)$ & $17(41.46)$ & 17 \\
\hline Pearson chi $^{2}(2)=41.5194 \quad p=0.000$ &
\end{tabular}

\section{Cost of dressings}

Cost of BLD and VGD analyzed using two-sample ttests showed that VGD was 4 times more expensive to use than BLD. Table 5 compares the mean cost in US dollars of the two types of dressing.
Table 5: Comparison of cost of dressing for BLD and VGD

\begin{tabular}{lcccr}
\hline $\begin{array}{l}\text { Type of } \\
\text { dressing }\end{array}$ & Frequency & $\begin{array}{l}\text { Mean cost } \\
\text { (USD) }\end{array}$ & SD & $95 \%$ CI \\
\hline BLD & 45 & 56 & 28.80 & 47.35 \\
& & & & 64.65 \\
VGD & 41 & 228.20 & 106.67 & 194.62 \\
& & & & 261.96 \\
Total & \multirow{2}{*}{138.14} & 115.22 & 113.44 \\
& & & & 162.84 \\
\hline Pr $(T<t)=0.0000 ;$ CI-confidence interval; SD standard dev- \\
deviation; USD-US Dollars
\end{tabular}

\section{Discussion}

A total of 86 patients were enrolled in the study: 52 males and 34 females; 45 patients were dressed with BLD and 41 with VGD. Discrepancies in the numbers enrolled for each arm of the study were due to loss to follow-up in a study where sampling was alternate.

\section{Postoperative pain}

Subjective postoperative pain scores revealed a reduction in pain on the SSG donor sites as days went by, as is expected in any wound that is healing normally. The postoperative pain trend was the same for both groups. Gore and Akolekar showed that BLD had a cooling effect on partial thickness burn wounds (8); similarly, in our study patients dressed with BLD were generally more comfortable and reported verbally that they had significant reduction in donor site pain than those with VGD. Patients who had the chance to undergo both dressings at different times, either from repeated surgeries or staged operations, reported higher pain scores on the VGD sites than on the BLD sites.

Analysis of the postoperative pain scores however did not reveal any significant relationship between the dressing applied and the pain scores. Therefore the pain patients experienced during the postoperative period might not have been necessarily influenced by the type of dressing applied on their SSG donor site wound but rather by other factors including age, sex, individual pain thresholds, among others, whose influence was not part of the objectives of this study. Severity of pain experienced upon dressing change on the 10th postoperative day showed that BLD patients had only mild to moderate pain compared with those dressed with VGD who reported severe pain. These pain scores can be attributed to the nature of the two dressings. As 
described by Gore and Akolekar (8), BLD has a large waxy non-adherent surface that does not integrate with underlying dermal and epithelial cells of the healing wound. In addition to allowing the wound to dry out, VGD integrates with the underlying epidermis, leading to trauma to the underlying epidermis during dressing change, hence increasing pain and bleeding. This is similar to what was found in studies by Queen and colleagues (9) and Jones (10). Patients who had BLD in this study only experienced severe pain if the BLD slipped off the wound, bringing the wound in direct contact with the overlying dressing or exposing it to air. This was seen in 3 patients with BLD in our study, and dressings had to be adjusted to cover the raw area of the wound.

\section{Wound re-epithelialization}

The rate of wound re-epithelialization was used as a measure of the rate of healing, and was shown to be faster in BLD than in VGD, similar to what Gore and Akolekar found (3). The faster rate may be attributed to certain properties of BLD that give it a better propensity for re-epithelialization. In our study, BLD was found to have a large smooth surface that provided occlusive dressing to the SSG donor site wounds and preserved moisture on the wound bed in addition to providing sufficient coverage for nearly all sizes of SSG donor site wounds. The ridged contour of BLD also allows excess exudate to drain from the wound surface into the surrounding absorbent dressing, without drying out the wound. In contrast, VGD allowed the wound surface to dry out both through evaporation and also through capillary absorbance of exudate from the wound into the overlying absorbent dressings. The importance of moisture in wound healing has been emphasized by Jones who stated that moisture allows epithelial migration (10). Similarly, Sagray and colleagues stated the need for moisture in the optimal healing of SSG donor sites (11). Another study by Bryan et al. stated that moist wounds had a higher concentration of macrophages by day 3 , fewer inflammatory cells by day 5 and were re-epithelialized by day 7 (12). Many studies alluding to the ideal wound dressing material have emphasized the need to maintain moisture balance at the wound-primary dressing interface (13), a property provided for by BLD.

\section{Microbial culture}

In our study, $40 \%$ of all patients had no organism grown on microbial culture, similar to what Rakel and colleagues concluded in their study, stating that SSG donor site infection was rare, occurring in only $5 \%$ of cases (14). A myriad of organisms however was isolated among the other $60 \%$ of the patients, the commonest being Staphylococcus aureus, a gram-positive coccus, which exists as normal flora on skin. The other organisms isolated occurred rarely and could be attributed to wound contamination or sample contamination during inoculation in the laboratory. One patient with BLD and one patient with VGD had signs of inflammation on their wounds on dressing change, which included erythema, swelling of surrounding skin and pus discharge. These could be attributed to infection, enhanced by individual patient factors, but occurred too rarely to be significant.

\section{Usability of dressings}

The use of BLD and VGD were compared in the ease of applying during intraoperative wound dressing, and ease of removing during dressing change on postoperative day 10. Subjective scores of eases of applying showed that both BLD and VGD were easy to apply. Application of BLD however required steady placement of absorbent dressings over the primary BLD dressing, taking caution to ensure the BLD does not slip and expose any part of the SSG donor site wound. The BLD dressing also had to be trimmed to allow only slight overlap over the SSG donor site wound edges, otherwise excess dressing covering normal skin wound causes friction between the BLD and skin on movement, causing friction burns and blistering of the normal skin and pain. This phenomenon was observed in 2 patients in this study.

On ease of removal, BLD was easier to remove than VGD. This is attributed to the smooth non-adherent surface of BLD, similar to what Gore and Akolekar found in their study (3). On the other hand, VGD undergoes progressive desiccation, adheres to the wound surface and may have epithelial cells growing over the gauze, causing the gauze to integrate with the 
underlying wound bed and increasing the difficulty of removing the dressing (9). Queen (9) also alluded to the pain and bleeding associated with VGD removal due to damage caused to the epithelium, and these properties of VGD are similar to what was found in our study.

\section{Cost of dressings}

BLD was found to be up to four times cheaper than VGD, making it a cheaper dressing suitable for regular use in a developing country, as recommended by Atiyeh et al. (15).

\section{Conclusion}

Banana leaf dressing, once placed and secured correctly, is an effective dressing for SSG donor sites as it is associated with less pain on dressing change, quickens epithelialization, does not predispose patients to any infection, and is relatively cheap compared with the traditional Vaseline gauze dressing.

\section{Limitations}

It was difficult to keep some participants admitted for the 10-day period of the study because of cost implications, hence the loss to follow-up of some patients. Scales used for estimating pain and for ease of use of dressings were subjective, compromising accuracy of results.

\section{Recommendations}

We recommend:

1. banana leaf dressings be adopted for more regular use on split thickness donor site wounds, burn wounds, and perhaps for general surgical wounds such as laceration and abrasions as well, because it is easy to remove.

2. banana leaf dressing be adopted by every hospital in Uganda and other low-income countries where bananas are available, because it is cheap.

3. another study to compare the use of banana leaf dressing with more recent dressing like Alginate dressings.

4. another study to examine the chemical components of banana leaf that may enhance its properties in hastening wound healing.

\section{References}

1. Masella PC, Balent EM, Carlson TL, et al. Evaluation of six split-thickness skin graft donor-site dressing materials in a swine Model. Plast Reconstr Surg Glob Open. 2013;1(9).

2. Demirtas Y, Yagmur C, Soylemez F, et al. Management of splitthickness skin graft donor site: a prospective clinical trial for comparison of five different dressing materials. Burns. 2010;36(7):999-1005.

3. Gore MA, Akolekar D. Banana leaf dressing for skin graft donor areas. Burns. 2003;29(5):483-6.

4. Kalyebara M, Ragama P, Kikulwe E et al. Economic importance of the banana bacterial wilt in Uganda. Afr Crop Sci J. 2006;14(2):93-103.

5. Guenova E, Hoetzenecker W, Kisuze G et al. Banana leaves as an alternative wound dressing. Derm Surg. 2013;39(2):290-7.

6. van Griensven H, Strong J, Unruh A. Introduction to pain. Pain: a textbook for health professionals. Edinburg, Churchill Livingstone. 2013:1-8

7. Cooper R. Ten top tips for taking a wound swab. Wounds International [Online]. Accessed November 2020. https://www.woundsinternational.com/resources/details/tentop-tips-for-taking-a-wound-swab.

8. Gore MA, Akolekar D. Evaluation of banana leaf dressing for partial thickness burn wounds. Burns. 2003;29(5):487-92.

9. Queen D, Orsted H, Sanada H, Sussman G. A dressing history. International wound journal. 2004;1(1):59-77.

10. Jones J. Winter's concept of moist wound healing: a review of the evidence and impact on clinical practice. J Wound Care. 2005;14(6):273-6.

11. Sagray BA, Lalani S, Mehan V. An alternative coverage for split thickness skin graft donor site wounds. J Foot Ankle Surg. 2011;50(3):369-71.

12. Bryan J. Moist wound healing: a concept that changed our practice. J Wound Care. 2004;13(6):227-8.

13. Bishop S, Walker M, Rogers A, et al. Importance of moisture balance at the wound-dressing interface. $J$ Wound Care. 2003;12(4):125-8.

14. Rakel BA, Bermel MA, Abbott LI, Baumler SK, et al. Splitthickness skin graft donor site care: a quantitative synthesis of the research. App Nurs Res. 1998;11(4):174-82.

15. Atiyeh B, Masellis A, Conte C. Optimizing burn treatment in developing low-and middle-income countries with limited health care resources (part 2). Ann Burns Fire Disasters. 2009;22(4):189-95. 